\title{
Is routine ureteral stenting really necessary after retrograde intrarenal surgery?
}

\author{
Ekrem Ozyuvali $^{1}$, Berkan Resorlu ${ }^{2}$, Ural Oguz $^{3}$, Yildiray Yildiz ${ }^{1}$, Tolga Sahin ${ }^{1}$, Cagri Senocak ${ }^{1}$, \\ Omer Faruk Bozkurt ${ }^{1}$, Erman Damar ${ }^{1}$, Murat Yildirim ${ }^{1}$, Ali Unsal ${ }^{4}$ \\ ${ }^{1}$ Department of Urology, Kecioren Training and Research Hospital, Ankara, Turkey; \\ 2 Department of Urology, Canakkale Onsekiz Mart University, Faculty of Medicine, Canakkale, Turkey; \\ ${ }^{3}$ Department of Urology, Giresun University, Faculty of Medicine, Giresun, Turkey; \\ ${ }^{4}$ Department of Urology, Gazi University, Faculty of Medicine, Ankara, Turkey.
}

\begin{abstract}
Summary Objectives: To investigate the situations in which ureteral double-J stent should be used after retrograde intrarenal surgery (RIRS). Patients and Methods: Patients with no ureteral double-J stent after RIRS constituted Group 1, and those with double-J stent after RIRS constituted Group 2. Patients' age and gender, renal stone characteristics (location and dimension), stone-free status, VAS score 8 hours after surgery, post-procedural renal colic attacks, length of hospitalization, requirement for re-hospitalization, time to rehospitalization and secondary procedure requirements were analyzed.

Results: RIRS was performed on 162 renal units. Double-J stent was used in 121 (74.6\%) of these after RIRS, but not in the other 41 (25.4\%). At radiological monitoring at the first month postoperatively after RIRS, complete stone-free status was determined in $122(75.3 \%)$ renal units, while residual stone was present in $40(24.6 \%)$. No significant differences were observed between the groups in terms of duration of fluoroscopy $(p=0.142)$, operation $(p=0.108)$ or hospitalization times $(p=0.798)$. VAS values determined routinely on the evening of surgery were significantly higher in Group 1 than in Group $2(p=0.025)$. Twenty-eight $(17.2 \%)$ presentations were made to the emergency clinic due to renal colic within 1 month after surgery. Double-J catheter was present in 24 (85.7\%) of these patients.
\end{abstract}

Conclusions: Routine double-J stent insertion after RIRS is not essential since it increases costs, morbidity and operation time.

KEY WORDS: Retrograde intrarenal surgery; Ureteral stent; Urolithiasis.

Submitted 26 September 2014; Accepted 30 November 2014

\section{INTRODUCTION}

Shock wave lithotripsy (SWL), ureteroscopy (URS) and percutaneous nephrolithotomy (PNL) are the most popular, minimally invasive options in the treatment of urinary system stone disease. Thanks to recent advances in holmium laser technology and the entry into use of new generation flexible ureteroscopes and miniature instruments, retrograde intrarenal surgery (RIRS) has now become a significant alternative in the treatment of renal stones (1). Many renal stones can today be effectively treated using this technique without the need for invasive methods such as PNL or open surgery. RIRS is particularly employed as a primary treatment method in stones smaller than $2 \mathrm{~cm}$ where SWL has been unsuccessful and in patients with skeletal deformities and bleeding diathesis (2).

While double-J stent following endourological surgical procedures is not routine, it is frequently employed for the purpose of preventing potential obstruction associated with postoperative ureteral edema or residual stone fragments (3). However, a wide range of complications, including infection, urinary complaints, hematuria, stent migration, encrustation and rupture can be seen in a significant proportion (10-85\%) of patients following double-J stent insertion (4). With the development of flexible ureteroscopes and miniaturization of calibration devices there has been a relative decline in the incidence of ureteral trauma, and routine double-J stent has become controversial $(3,4)$. Although there have been various studies concerning the use of double-J stent after rigid URS, there are no data in the literature regarding the situations in which double-J stent should be used post-RIRS (5). Use of ureteral access sheaths during RIRS and the frequent observation of complications such as postoperative renal colic and urosepsis differentiate this technique from rigid operations. This study investigated the situations in which double-J stent should be used post-RIRS.

\section{Patients AND methods}

One hundred fifty-six patients (162 renal units) diagnosed with renal stone, who underwent RIRS at our clinic between January 2011 and June 2012 and met the inclusion criteria, were included in the study. Seventy-nine patients (50.56\%) were men and 77 (49.4\%) women, with a mean age of 39.4 years (2-82). Seventy-three stones (45\%) were located in the right renal unit and $83(51.2 \%)$ 
Table 1.

Patient and stone characteristics.

\begin{tabular}{|l|c|}
\hline No. of patients (renal unit) & $156(162)$ \\
\hline Mean age (year) & $39.4(2-82)$ \\
\hline Male/female & $79 / 77$ \\
\hline Stone side (\%) & $83(51 \%)$ \\
Left & $73(45 \%)$ \\
Right & $6(3.7 \%)$ \\
$\quad$ Bilateral & $98(60.4 \%)$ \\
\hline Stone location (\%) & $64(39.6 \%)$ \\
$\quad$ Renal pelvis & $14(5-35)$ \\
Calyx & $1.3(1-3)$ \\
\hline Mean stone size (mm) & \\
\hline Mean stone number & \\
\hline
\end{tabular}

in the left. Bilateral renal stones were present in 6 patients (3.7\%). Stone dimensions and the longest axis for each stone were calculated. Mean stone size was $14.6 \mathrm{~mm}$ (5$35 \mathrm{~mm})$ and mean stone number 1.3. Patients' demographic characteristics are shown in Table 1.

Patients with stones smaller than $2 \mathrm{~cm}$ (total $35 \mathrm{~mm}$ in multiple stones), obese patients, patient with skeletal deformity or bleeding diathesis, patients with renal stones requiring complete removal (repeating infection, pilots, etc.) and subjects with stone in which SWL treatment had been ineffective were included. Patients with a pre-diagnosis of tumor, with a diagnosis of ureteral stricture, with severe mucosal injury or ureteral perforation arising during surgery and patients with a solitary kidney were excluded. Complete blood count, blood biochemistry, coagulation parameters, ELISA tests and urine tests and culture analyses were investigated pre-operatively in all patients. Patients with growth in culture were given the appropriate antibiotic therapy, and surgery was planned once urine was sterile. All patients scheduled for surgery after diagnosis of renal stone were assessed with preoperative contrast examination (intravenous urography [IVU] or computerized tomography [CT]) if this had not been performed previously and was not contraindicated (allergy, pregnancy, etc.).

\section{Surgical technique}

All operations were performed under general anesthesia and with the patient in the dorsal lithotomy position. An $8 \mathrm{~F}$ feeding tube was inserted into the bladder by the urethral path in order to avoid perioperative bladder filling. A 7.5 F Storz Flex-X2 was used for flexible URS. A guide wire was first inserted into the ureter under fluoroscopic monitoring, and the ureteral access sheath was then pushed forward as far as the renal pelvis. The guide wires used were coated in polytetrafluorethylene (PTFE) or hydrophilic material, and ranged between 80 and $260 \mathrm{~cm}$ in length and 0.035 or 0.038 inch in diameter. The ureteral access sheaths used had an external diameter of 9.5-14 F and were 35-55 cm in length. Double open ended 4F, 4.8F, or 6F catheters were used for double-J stent. Catheters were inserted into the ureter with the help of a guide wire. Patients with no pain or fever were discharged on the 1st day postoperatively. Stone clearance was assessed intraoperatively by direct URS and postoperatively by imaging. All patients underwent ultrasonog- raphy (US) and radiographic study examination on a routine basis the day after RIRS to ensure the absence of hydronephrosis and stone. Treatment success was defined as stone-free or clinically insignificant residual fragments (residual fragment $<3 \mathrm{~mm}$ ).

Double-J Stents were removed from patients in 2-4 weeks postoperatively. Before stent removal, patients were monitored radiologically for presence of stone.

Patients with no ureteral double-J stent after RIRS constituted Group 1, and those with double-J stent after RIRS constituted Group 2. Patients' age and gender, renal stone characteristics (location and dimension), stone-free status, VAS score $8 \mathrm{~h}$ after surgery, post-procedural renal colic attacks, length of hospitalization, requirement for re-hospitalization, time to re-hospitalization and secondary procedure requirements were analyzed. Categoric variables were expressed as numbers and percentages and constant variables as means. Categoric variables between groups were compared using the chi square test. The MannWhitney $U$ test was used to examine differences between groups after normality testing. Data obtained were analyzed on SPSS 15.0. Significance was set at $\mathrm{P}<0.05$.

\section{RESULTS}

RIRS was performed on 162 renal units. Double-J stent was used in 121 (74.6\%) of these post-RIRS, but not in the other 41 (25.4\%). Mean stone dimension was 14.6 mm (5$35 \mathrm{~mm}$ ) and mean stone number was 1.3 (1-3). Stone was located in the renal pelvis in 98 (60.4\%) renal units and in the calyx in 64 (39.6\%). Mean length of surgery was 37.2 minutes (15-140). Mean duration of scope was $39 \mathrm{sec}(0-$ $140 \mathrm{sec})$ and mean length of hospitalization was 1.8 days (1-8 days). The results are summarized in Table 2.

At radiological monitoring at first month postoperatively post-RIRS, complete stone-free status was determined in $122(75.3 \%)$ renal units, while residual stone were present in 40 (24.6\%). One renal unit with more than one stone achieved stone-free status using PNL on the 5th day. Stone-free status was achieved with URS in four patients one month postoperatively and in six patients by repeating RIRS. Five patients were referred for SWL with insertion of a double-J stent. The other cases did not continue with the protocol.

Mean stone size in the double-J stent group was $15 \mathrm{~mm}$,

\section{Table 2.}

Comparison of groups according to gender, stone location, SWL history, success and re-hospitalization rates.

\begin{tabular}{|llcccc|}
\hline \multicolumn{1}{|c}{ Gender } & Group 1 & Group 2 & Total & P value \\
\hline & Female & $24(58 \%)$ & $57(47 \%)$ & $81(50 \%)$ & 0.206 \\
Stone location & $172 \%)$ & $64(53 \%)$ & $81(50 \%)$ & \\
& Pelvis & $16(39 \%)$ & $82(68 \%)$ & $98(60 \%)$ & $0.002 *$ \\
& Calyx & $25(60 \%)$ & $39(32 \%)$ & $64(40 \%)$ & \\
\hline Previous SWL & Yes & $25(60 \%)$ & $68(56 \%)$ & $93(57 \%)$ & 0.725 \\
\hline Success & $78 \%$ & $74 \%$ & $75 \%$ & 0.794 \\
\hline Re-Hospitalization & $4(9 \%)$ & $24(19 \%)$ & $28(17 \%)$ & 0.216 \\
\hline * Statistically Significant At $P<0.05$. & & & \\
\hline
\end{tabular}


Table 2.

Patient demographics, stone characteristics and operative findings of groups

\begin{tabular}{|ll|c|c|c|c|c|c|}
\hline & & Mean & Median & Min & Max & SS & P value \\
\hline Age (years) & Group 1 & 35 & 37 & 2 & 76 & 16,18 & \\
& Group 2 & 40 & 42 & 2 & 82 & 18,03 & \\
& Total & 39 & 40,5 & 2 & 82 & 17,64 & 0.123 \\
\hline Stone size (mm) & Group 1 & 13 & 12 & 5 & 30 & 5,92 & \\
& Group 2 & 15 & 15 & 6 & 35 & 5,22 & \\
& Total & 14 & 14 & 5 & 35 & 5,43 & $0.019 *$ \\
\hline Stone number & Group 1 & 1,27 & 1 & 1 & 3 & 0,55 & \\
& Group 2 & 1,31 & 1 & 1 & 6 & 0,73 & \\
& Total & 1,30 & 1 & 1 & 6 & 0,69 & 0.998 \\
\hline Operation time (min) & Group 1 & 33 & 32 & 18 & 65 & 11,27 & \\
& Group 2 & 38 & 36 & 15 & 140 & 16,73 & \\
& Total & 37 & 35 & 15 & 140 & 15,63 & 0.108 \\
\hline Fluoroscopy (sec) & Group 1 & 37 & 24 & 0 & 140 & 36,27 & \\
& Group 2 & 39 & 35 & 0 & 132 & 26,68 & \\
& Total & 39 & 34,5 & 0 & 140 & 29,29 & 0.142 \\
\hline Hospitalization time (days) & Group 1 & 1,6 & 2 & 1 & 3 & 0,61 & \\
& Group 2 & 1,8 & 2 & 1 & 7 & 1,02 & \\
& Total & 1,8 & 2 & 1 & 7 & 0,93 & 0.798 \\
\hline VAS level & Group 1 & 5,9 & 6 & 2 & 10 & 1,86 & \\
& Group 2 & 5,1 & 5 & 2 & 10 & 1,88 & \\
& Total & 5,3 & 5 & 2 & 10 & 1,90 & 0.025 \\
\hline * Statistically significant at $p<0.05$. & & & & & \\
& & &
\end{tabular}

lithotriptor effectiveness have led to a decrease in the use of stents. While stent use is necessary after traumatic procedures, some Authors suggest that routine stent insertion is not necessary postprocedurally $(7,8)$. Complication retes in the literature range from $5 \%$ to $10 \%$, with a rate of major complications of approximately 1\% (9). Ureteral avulsion continues to represent a major acute complication. Complications are strongly correlated with equipment used and urologist experience $(6,7,9)$. In our case, ureteral perforation during ureteral sheath insertion developed in two renal units. The procedure in these patients was concluded with double-J stent insertion. No findings of extravasation or urinoma were detected at postoperative follow-up period.

The purpose of stent insertion after ureteroscopic lithotripsy is to prevent ureteral stricture, achieve healing and to facilitate passage of stone fragments in the ureter with passive dilation $(10,11)$. Stent is required after URS in the event

compared to $12 \mathrm{~mm}$ in the group without stent ( $\mathrm{p}=$ 0.019). No significant difference was observed between the groups in terms of stone number $(p=0.098)$. A significant rate of the stones in both groups were located in the renal pelvis ( $\mathrm{n}=98,60.4 \%$ ). A significantly higher proportion of stones were located in the renal pelvis in Group 2 than in Group 1 ( $p=0.002)$. In addition, VAS values determined routinely on the evening of surgery were significantly higher in Group 1 than in Group $2(p=0.025)$. No significant difference was observed between the groups in terms of age $(p=0.123)$ or gender $(p=0.206)$.

No significant differences were determined between the groups in terms of duration of fluoroscopy $(p=0.142)$, operation ( $p=0.108)$ or hospitalization times $(p=0.798)$. Ureteral perforation was observed as a complication in two patients. These were treated with insertion of a double-J stent. No extravasation or stricture pattern was observed at IVU performed in the $3^{\text {rd }}$ month postoperatively, and stone-free status was subsequently achieved using repeat RIRS. Urosepsis was observed in one case in Group 2. This patient was treated with antibiotic therapy under intensive care conditions. Urinary tract infection was observed in 23 cases (14.1\%) within the 1st month postoperatively. These were treated on the basis of culture antibiogram results. Twenty-eight (17.2\%) presentations were made to the emergency clinic due to renal colic within 1 month after surgery. Double-J catheter was present in $24(85.7 \%)$ of these patients. No major complication such as avulsion, iatrogenic organ injury, urinoma or hydropneumothorax developed in any renal unit (Table 3).

\section{Discussion}

Stents have been used in the treatment of kidney and ureter stones for more than 30 years (6). Development of fine callibration ureteroscopes and improvements in of mucosal edema, epithelium injury, mucosal bleeding and ureteral perforation and in patients with solitary kidney. Stents can also be inserted for the purpose of preventing potential complications in patients undergoing ureteral lower tip dilation before URS. Stent insertion has been shown to reduce temporary lower urinary system pain and symptoms (12). Renal colic develops within the first 24 hours in patients not using stent. When injury develops during RIRS, 48-72 hours are needed to elapse for edema to develop, and it is therefore incorrect to attribute renal colic attacks in the first 24 hours to edema alone. Residual stone fragments, coagulum or ureteral spasm or both are among the possible causes (13).

Tanriverdi et al. reported that emergency double-J stent insertion in the early postoperative period (first 24 hours) in 23 patients with uncompleted URS prevented progressive obstruction and potential post-surgical complications (14).

However, there are some disadvantages of stents, including urinary tract infection, dysuria, pollakiuria, hematuria and need for repeated cystoscopy for stent migration or stent extraction. Some Authors are of the opinion that stent insertion increases costs by prolonging length of surgery and due to cystoscopy requirement for stent removal. The stent removal procedure has also been reported to be traumatic (15).

Byrne et al. reported that stent insertion increased length of surgery by $12 \mathrm{~min}$ (16). Netto et al. reported a length of surgery of 65 min in a group with stent insertion and 45 min in patients without stenting (17). Mean duration of surgery in the 41 renal units without stent insertion in our study was $33.6 \mathrm{~min}$ (18-65 min), compared to 38.4 min (15-140) in the 121 renal units with stenting (15$140 \mathrm{~min}$ ). The difference was not significant ( $p>0.05$ ) but no cost analysis was performed in our study. Some studies evaluating complication rates associated with 
stent use have reported higher levels of complications in the group without stent insertion $(15,18)$.

The fact that double-J stent was inserted in 121 renal units although a $7.5 \mathrm{~F}$ flexible ureteroscope was used in our study group conflicts with existing URS data. However, those studies were performed for rigid URS and generally used a pneumatic lithotriptor. The studies performed with RIRS/holmium;YAG lasers are insufficient. In our study, renal colic attacks were observed in 78 patients (64\%) of the group receiving double-J stents post-procedurally and in 24 (58\%) of the group not receiving double-J stent with secondary double-J stent insertion required in 16 of these. However, no statistically significant difference was determined regarding this finding $(p=0.239)$. The incidence of renal colic attacks and number of double-J insertions being greater than those in the literature may be attributed to the use of a ureteral sheath during RIRS and probably to ureteral edema. In addition, working under constant positive hydrostatic pressure in order to overcome the inadequacy of flexible URS in providing an optimal image of a narrow channel during RIRS can trigger renal colic attacks and infection leading to fornix rupture and pyelolymphatic and pyelo-renal backflow.

No significant differences were determined in our study in terms of age, gender, stone number, history of surgical intervention before the procedure, history of SWL, length of surgery, length of scope use, renal colic, analgesic requirement and duration of hospitalization, and double-J stent requirement $(p>0.05)$.

The guidelines generally state that ureteral stent insertion is not necessary after URS performed for stone without complications. However, there are reports in the current literature of increasing double-J stent requirement due to increasing incidence of ureteral trauma related to use of the ureteral access sheath (7).

\section{Conclusion}

Routine double-J stent insertion after RIRS is not essential since it increases costs, morbidity and operation time. Double-J stent insertion in stones larger than 15 $\mathrm{mm}$ located in the renal pelvis is positively and significantly correlated with VAS score $(p<0.05)$. We think that further studies with wider case series and long-term follow-up are needed to better assess this topic.

\section{References}

1. Akincr M, Esen T, Tellaloglu S. Urinary stone disease in Turkey: an update epidemiological study. Eur Urol. 1991; 20:200-3.

2. Resorlu B, Unsal A. Retrograde Intrarenal Surgery (RIRS) for Renal Stones. Turk Urol Sem. 2011; 2:64-7.

3. Ates F, Adayener C, Akyol I, et al. Üreteroskopik Litotripsi Sonrasi Her Zaman Üreteral Stent Yerlestirmek Gerekir mi? Bakırköy Tıp Dergisi. 2010; 6:142-7.

4. Geavlete P, Georgescu D, Nita G, et al. Complications of 2,735 retrograde semirigid ureteroscopy procedures: a single-center experience. J Endourol. 2006; 20:179-85.

5. Resorlu B, Unsal A, Gulec H, et al. A new scoring system for predicting stone-free rate after retrograde intrarenal surgery: the 'Resorlu-Unsal stone score'. Urology. 2012; 80:512-8.
6. Johnson DE, Cromeens DM, Price RE. Use of the holmium:YAG laser in urology. Lasers Surg Med. 1992; 12:353-63.

7. Sayer J, Johnson DE, Price RE, et al. Ureteral lit-hotripsy with the holmium:YAG laser. Laser Med. 1993; 11:61-5.

8. Alan C, Koçoglu H, Ersay AR. Retrograde itntrarenal surgery: technic, clinical results, tips and tricks, The New Journal of Urology. 2011; 6:32-41.

9. Conlin MJ Ureteropyeloscopy for calculi In Nakada SY, Pearle MS. (eds) Advanced Endourology, Humana Press Inc, Totowa New Jersey 2006, pp 105-108.

10. Atug F, Akay F, Akkus Z, et al. Komplike olmayan üreteroskopik litotripsilerden sonra üreteral stent yerlestirilmesi gerekli midir? Türk Üroloji Dergisi. 2006; 32: 225-9.

11. Tepeler A, Resorlu B, Sahin T, et al. Categorization of intraoperative ureteroscopy complications using modified Satava classification system. World J Urol. 2014; 32:131-6.

12. Damiano R, Oliva A, Esposito C, et al. Early and late complications of double pigtail ureteral stent. Urol Int. 2002; 69:136-40.

13. Keeley FX Jr, Timoney AG. Routine stenting after ureteroscopy: think again. Eur Urol. 2007; 52:642-4.

14. Tannverdi O, Yencilek F, Koyuncu H, et al. Emergent Stenting After Uncomplicated Ureteroscopy. Urology. 2011; 77:305-8.

15. Borboroglu PG, Amling CL, Schenkman NS, et al. Ureteral stenting after ureteroscopy for distal ureteral calculi: a multi-institutional prospective randomized controlled study assessing pain, outcomes and complications. J Urol. 2001; 166:1651-7.

16. Byrne RR, Auge BK, Kourambas J, et al. Routine ure-teral stenting is not necessary after ureteroscopy and ureteropyeloscopy: a randomized trial. J Endourol. 2002; 16:9-13.

17. Netto NR Jr, Ikonomidis J, Zillo C. Routine ureteral stenting after ureteroscopy for ureteral lithiasis: is it really necessary? J Urol. 2001; 166:1252-4.

18. Joshi HB, Stainthorpe A, MacDonagh RP, et al. Indwelling ureteral stents: evaluation of symptoms, quality of life and utility. J Urol. 2003; 169:1065-9.

\section{Correspondence}

Ekrem Ozyuvali, MD

Yildiray Yildiz, MD

Tolga Sahin, MD

Cagri Senocak, MD

Omer Faruk Bozkurt, MD

Erman Damar, MD

Murat Yildirim, MD

Department of Urology, Kecioren Training and Research Hospital, Ankara, Turkey

Berkan Resorlu, MD (Corresponding Author)

drberkan79@gmail.com

Canakkale Onsekiz Mart Universitesi, Terzioglu Yerleskesi, Barbaros Mh, 17100, Canakkale, Turkey

Ural Oguz, MD

Department of Urology, Giresun University, Faculty of Medicine, Giresun, Turkey

Ali Unsal, MD

Department of Urology, Gazi University, Faculty of Medicine, Ankara, Turkey 